\title{
The Sequential Compound Option Pricing with Random Interest Rate and Application to Project Valuation
}

\author{
Meng-Yu Lee ${ }^{\mathrm{a},}$ Fang-Bo Yeh $^{\mathrm{b}}$ An-Pin Chen ${ }^{\mathrm{a}}$ \\ a: Institute of Information Management, National Chiao-Tung University, Taiwan. mongyu@iim.nctu.edu.tw; \\ b: Department of Mathematics, Tunghai University, Taiwan. f.b.yeh@math.thu.edu.tw
}

\begin{abstract}
This paper proposes the pricing formula of sequential compound options (SCOs) with random interest rate and the applications call Milestone Project Valuation (MPV). Most compound options in literatures are 2 -fold with constant parameters through time. The multi-fold compound options are just sequential compound CALL options. The multi-fold sequential compound options proposed in this study are compound option on (compound) option with random interest rate and allow call/put alteration. Besides, the parameters can vary in different folds and make the model more flexible. The SCOs can enhance and broaden the usages of compound option in real option and financial derivative fields, including MPV. The projects that set some critical milestones, which should be achieved sequentially, are called milestone projects. This study propose the milestone project valuation by SCOs with random interest rate.
\end{abstract}

Keywords: sequential compound option, project valuation, real option, random interest rate, option pricing

\section{Introduction}

Compound options, the options with options as underlying, are one of the important financial innovations. The fold number of a compound option counts how many option layers tacking directly on other underlying options. Original compound options are proposed by Geske (1979) with 2-fold. A specific multi-fold compound option formula is revealed by Carr (1988) while the sequential compound call (SCC) is proved by Thomassen \& Van Wouwe (2001) and Chen (2003). Chen (2002) and Lajeri-Chaherli (2002) prove the 2-fold compound options through risk-neutral method simultaneously. Elettra \& Rossella (2003) generalized the 2 fold compound calls by time-dependent parameters.

Many financial applications extending from the compound option theory are widely employed. The seminal study by Geske and Johnson (1984a) derived the analytic American put option under the inspiration of compound option, while Carr (1988) presented the sequential exchange options formula. Corporate debt (Geske \& Johnson, 1984b; Chen, 2003), chooser options (Rubinstein, 1992), capletions and floortions (the options of the interest rate options) (Musiela \& Rutkowski, 1998) are also priced by compound options.

Besides the financial derivative pricing, the compound option theory is also used widely in the real option field originated by Trigeorgis (1993). However, the sophisticated structure of the derivative pricing and the wide deployment in the real option field make the current compound option methodology insufficient. The 2-fold compound options are not enough for the block-building financial innovations whereas the multi-fold compound options focus on the sequential compound calls only.

This paper extend the SCOs from fold-wise interest rate (Lee et al., 2006) to random interest rate.
The SCOs use vanilla European options as building blocks and extend the compound option theory to multi-fold sequential compound options with random interest rate as well as (SCOs) alternating puts and calls. The SCOs are (compound) options on compound options, where the option features of different folds could be assigned arbitrarily as call or put. The SCOs presented in this paper allow the parameters (such as volatility) to vary in different folds. The random interest rate model derived by the forward measure enable the long-term SCOs more realistic. The explicit valuation formula and sensitivity analysis of SCOs are proved by the risk-neutral method in this study. Comparing with the P.D.E. method, there is more financial intuition coinciding with the risk-neutral SCOs derivation.

The multi-fold SCOs alternating puts/calls with fold-dependent parameters can enhance the compound option application, especially in real option fields. The real world cases may often be multiple interacting options containing different option types (Trigeorgis,1993), such as expansion, contraction, shutting down, abandon, switch and or growth. The interaction between different types of options could be evaluated by the SCOs. The SCOs proposed in this study make the exotic multiple interacting option valuation possible. Also, the financial derivative pricing, such as exotic chooser options and capletions, can also employ the SCOs.

The applications of SCOs call Milestone Projection Valuation (MPV) is proposed in this paper. The projects that set some critical milestones, which should be achieved sequentially, are called milestone projects. The milestone projects would fail if any one of the serial milestones is not completed. The MPV method is designed for multi-stage project based on the results of SCO. Each milestone completion has the choice to enter the next stage or not, hence the sequential project milestone could be viewed as the sequential compound CALL options.

This paper is arranged as the following: section 2 presents the SCOs pricing formula. Section 3 exhibits the MPV method. The paper ends with the conclusion.

\section{Sequential Compound Options with Random Interest Rate}

This section defines the notations and derives the multi-fold SCOs with interest rate through the forward measure method. The SCOs, composed of European options, are the (compound) option on compound options, where the option features of different folds could be assigned arbitrarily as either call or put.

Denote the correlation matrix $\mathbf{Q}_{k}:=\left[Q_{\{k, g, h}\right]_{k \times k}$, where $Q_{\{k,, g, h}$ is the symmetric $(g, h)$ entry of the $\mathbf{Q}_{k}$, $\forall 1 \leq g \leq h \leq k$. Similarly, $d_{\{k\}, g}$ is the $g$-th entry of the vector $\left[d_{i k, g, g}\right]_{\times 1} \cdot\left(\left[Q_{i k i, g, h}\right]_{k \times k}\right)^{-i,-j)}$ is the $(k-1)$ by $(k-1)$ matrix which exclude the $i$ row and the $j$ column of $\left[Q_{k k, g, n}\right]_{k \times k}$. Denote the function $f(z)=\frac{1}{\sqrt{2 \pi}} e^{-\frac{1}{z^{2}}}$. The 
$k$-variate normal integral with upper bound limit vector $\left[d_{\{k\}, g}\right]_{x 1}$ and correlation matrix $\mathbf{Q}_{k}$ is charactered as

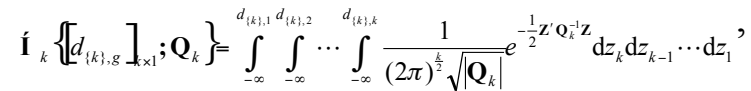
where $\mathbf{Z}^{\prime}=\left[z_{1}, z_{2}, \cdots, z_{k}\right], \mathbf{N}_{0} \equiv 1$.

Curnow $\&$ Dunnett (1962) reveals that the $k$-variate normal integral can be constructed from the $(k-1)$-variate by adding another dimension to the upper limit vector and correlation matrix.

Assume $T_{u-1}<T_{u}, \forall u \geq 1$. For any time interval from $T_{u-1}$ to $T_{u}(u \geq 1)$ with interval size $\tau_{u}$, its annualized volatility of asset price of this period are $\sigma_{u}^{2}$. Assume the volatility of forward price, $\sigma(t)$, is fold-wise constant, $\sigma(t)=\sigma, \forall T_{i-1}<t \leq T$. Denote the asset price at time $T_{u}$ as $S_{u}$. Denote the interest rate process $r(t)$ $T \leq t \leq T$ and the discount process $D(t)=e^{\tau_{0}}$. Let $B(t, T)$ be the zero coupon bond price at time $t$ that matures at time $T$. Denote the $\tau$-forward price of $T$-maturing zero coupon price at time $t$ as $F(t, \tau, T)$, $\forall t \leq \tau \leq T$.

Denote $\Psi^{\circledast}(T)$ as the random interest rate $i$-fold SCO price with strike $K_{1}$. This SCO starts at time $T_{0}$ and expires at time $T_{1}$. Its underlying asset is the $(i-1)$-fold SCO, $\Psi_{i-1}^{\circledast}\left(T_{1}\right)$, active from $T_{1}$ to $T_{2}$. Hence, the underlying SCO with fold number $(i-u+1), \quad \Psi_{i-u+1}^{\circledast}\left(T_{u-1}\right)$, validates from $T_{u-1}$ to $T_{u}$ with strike $K_{u}$ and parameters $\sigma^{2}$ under the assumption that the last fold SCO starting from $T_{0}$. The first fold option, $\Psi_{1}^{\circledast}\left(T_{i-1}\right)$, is a vanilla option with the asset as its underlying. It should note that fold numbers come in the reverse order.

The option feature, $\Lambda_{u, u}$, represents the call or put attribute of the (underlying) SCO with fold number (i-u+1) ranging from $T_{u-1}$ to $T_{u}, \forall u \geq 1$. If the SCO of this fold is a call, $\Lambda_{u, u}=1$; otherwise, $\Lambda_{u, u}=-1$ for the put. For example, a call on a put (2-fold compound option) starting at $T_{0}$ has option features with $\Lambda_{1,1}=1$ and $\Lambda$ ${ }_{2,2}=-1$. Denote $\Lambda_{h, g}={ }_{u=0}^{h} \Lambda_{u, \nu}, \forall 1 \leq g \leq h$, and $\Lambda_{1,0} \equiv 1$.

Under the same assumption of Thomassen and Van Wouwe (2001) except "parameters constant in each fold", the following theorem derives the pricing formula of the $i$-fold SCO with random interest rate alternating calls and puts by the risk-neutral method. Although the SCO presented in later section can start at any time $T_{u}$, the SCO in this theorem is starting from $T_{0}$ without loss of generality. The notation " *v", meaning "start from time $T_{v}{ }^{\prime \prime}$, is designed for time shift in sensitivity derivation. Under the above notations, the $i$-fold SCO price at time $T_{0}, \Psi_{i}^{\circledast}\left(T_{0}\right)$, is represented in Theorem 1 .

Theorem 1: Sequential compound option pricing with random interest rate

Denote

(1) $a_{i, g}^{\circledR}=\frac{\ln \left(\frac{F\left(T_{0}, T_{0}, T_{g}\right)}{\left.S_{\# g, i}^{\circledR}\right)+\frac{1}{2} \sum_{u=1}^{g} \sigma_{u}^{2} \tau_{u}}\right.}{\sqrt{\sum_{u=v+1}^{v+g} \sigma_{u}^{2} \tau_{u}}}, \quad \forall g \geq 1$

(2) $b_{i, g}^{\circledast}=a_{i, g}^{\circledast}-\sqrt{\sum_{u=1}^{g} \sigma_{u}^{2} \tau_{u}}, \quad \forall g \geq 1$

(3) $\hat{\rho}_{g, h}=\Lambda_{h-1, g} \rho_{g, h}, \forall h>g \geq 1 ; \rho_{g, g}=1, \forall g$;

$\rho_{g, h}=\rho_{h, g}, \forall h, g ; \rho_{g, h}=\sqrt{\sqrt{\frac{\sum_{i=1}^{g} \sigma_{u}^{2} \tau_{u}}{u=1} \sigma_{u}^{2} \tau_{u}}}, \forall 1 \leq g<h$.

(4) Equivalent asset price (EAP)

$S_{\# g, i}^{\circledast}=\left\{\begin{array}{l}K_{i}, \text { for } g=i \\ \text { The stock price which makes } \emptyset_{i-g}^{\circledast}\left(T_{g}\right)=K_{g}, \forall 1 \leq g<i\end{array}\right.$ then

$\left.\Psi_{i}^{\circledast}\left(T_{0}\right)=\Lambda_{i, 1} S_{0} \dot{\mathbf{I}} ;\left\{\left\lfloor\Lambda_{i, g} a_{i, g}^{\oplus}\right\rfloor_{\times 1} ; \mid \hat{\rho}_{g, h}\right\rfloor_{\times i}\right\}$

$\left.-\sum_{j=1}^{i} \Lambda_{j, 1} B\left(T_{0}, T_{j}\right) K_{j} \mathbf{I} ;\left\{\Lambda_{i, g} b_{i, g}^{\circledast}\right]_{\times 1} ;\left[\hat{\rho}_{g, h}\right]_{\times j}\right\}$

with assumptions that the $\operatorname{EAP}\left(S_{\# g, i}^{\circledR}\right)$ exists, $\forall 1 \leq g \leq i$.

Pf:

This theorem is proved by induction. The dynamics of related securities are exhibited before the induction procedures. Let $T$ be a fixed maturity date and $\widetilde{P}$ be the risk-neutral measure. Let $E[\cdot]$ be the expectation operator under $P$. Given $\tau$ satisfying $T_{0} \leq \tau \leq T$, let $\widetilde{P}^{\tau}$ be the $\tau$-forward measure, that is,

$d \widetilde{P}^{\tau}=\frac{D(T)}{B\left(T_{0}, \tau\right)} d \widetilde{P}$, where $B\left(T_{0}, \tau\right)=\widetilde{E}[D(\tau)]$.

Under $\widetilde{P}^{T}$, it is known that the forward price, $F(t, \tau, T)$, set at time $T_{0}$ for an investment starting at time $\tau$ and yielding one unit of currency at time $T$, is a martingale, for $T \leq t \leq \tau$. Let $\widetilde{W}^{\tau}$ be the Brownian Motion under $\widetilde{P}^{\tau}$. Assume that the dynamics of the forward price is

$d F(t, \tau, T)=\sigma(t) F(t, \tau, T) d \tilde{W}_{t}^{\tau} \quad$.By Ito Lemma,

$F(t, \tau, T)=F(T, \tau, T) e^{\left\{-\frac{1}{2} \int_{\tau_{0}}^{t} \sigma^{2}(u) d u+\int_{\tau_{0}}^{\sigma(u) d d \tilde{W}} \tau\right\}} \ldots \ldots(2.2)$

Take the asset price $S(\tau)$ as the numéraire. Under $\widetilde{P}^{\tau} \quad, \quad \frac{B(\tau, T)}{S(\tau)}=\frac{1}{F(t, \tau, T)} . \quad$ By $\quad$ Ito Lemma, $d\left[\frac{1}{F(t, \tau, T)}\right]=\frac{\left.-\sigma(t) d d \widetilde{W}_{t}^{\tau}-\sigma(t) d t\right]}{F(t, \tau, T)}$, for $T_{0} \leq t \leq \tau$. Let

$$
d \tilde{W}_{t}^{s}=d \tilde{W}_{t}^{\tau}-\sigma(t) d t .
$$

By Girsanov's Theorem, as $d \widetilde{P}^{S}$ satisfies $d \widetilde{P}^{s}=\frac{D(T) S(T)}{S\left(T_{0}\right)} d \widetilde{P}^{\tau}, \widetilde{W}_{t}^{s}$ is the Brownian Motion under $\widetilde{\boldsymbol{P}}^{s}$. Hence,

$d\left[\frac{1}{F(t, \tau, T)}\right]=\frac{-\sigma(t)\left\lfloor d \tilde{W}_{t}^{\tau}-\sigma(t) d t\right.}{F(t, \tau, T)}=\frac{-\sigma(t) d \tilde{W}_{t}^{s}}{F(t, \tau, T)}$ is a martingale under $\tilde{\boldsymbol{P}}^{s}$. By Ito Lemma again, $d \ln \left[\frac{1}{F(t, \tau, T)}\right]=-\frac{1}{2} \sigma^{2}(t) d t-\sigma(t) d \tilde{W}_{t}^{s}$. Thus,

$\frac{1}{F(t, \tau, T)}=\frac{1}{F(0, \tau, T)} e^{\left\{-\frac{1}{2} \int_{\tau_{0}^{2}(u) d u+\int_{\tau_{0}}^{\sigma}(u) d \tilde{W}_{u}^{s}}^{t}\right\}}$.

The exploited dynamics are used for the induction. The (2.1) is true for $i=1$. For the case $\Lambda_{1,1}=+1$ is exhibited in Shreve (2004) and the other case, $\Lambda_{1,1}=1$, can be proved by the similar way.

Assume the (2.1) is true for the $i$-fold compound option $\Psi^{\circledast}\left(T_{0}\right)$, it is showed that the (2.1) is also true for the $i+1$-fold compound option, for any $\Lambda_{g, g}, 1 \leq g \leq i+1$.

Because the underlying asset of $\Psi^{\otimes}\left(T_{0}\right)$ is $\Psi_{i}^{\circledast}\left(T_{1}\right)$, instead of $\Psi_{i}^{\circledast}\left(T_{0}\right)$, the start time of the $i$-fold compound option is shift from $T_{0}$ to $T_{1}$. All the notations of the $i$-fold compound option are changed simultaneously according to the time shift. Hence

$$
\begin{aligned}
& \left.\Psi_{i}^{\circledR}\left(T_{1}\right)=\Lambda_{i+1,2} S_{1} \dot{\mathbf{I}}_{i}\left\{\Lambda_{i+1, g+1} a_{i, g, * 1}^{\circledast}\right\rfloor_{1} ;\left[\hat{\rho}_{g, h, *_{1}}\right]_{\times i}\right\} \\
& -\sum_{j=1}^{i} \Lambda_{j+1,2} B\left(T_{1}, T_{j+1}\right) K_{j+1} \dot{\mathbf{I}}_{j}\left\{\left\lfloor\Lambda_{i+1, g+1} b_{i, g, *_{1}}^{\circledast}\right\rfloor_{\times 1} ;\left\lfloor\hat{\rho}_{g, h,{ }^{* 1}}\right\rfloor_{\times j}\right\}
\end{aligned}
$$

At the maturity time $T_{1}$ of the $i+1$-fold compound option, the price $\Psi_{i+1}^{\circledast}\left(T_{1}\right)=\max \left[\Lambda_{1,1} \Psi_{i}^{\circledast}\left(T_{1}\right)-\Lambda_{1,1} K_{1}\right]$; at 
the start time $T_{0}$, the option price

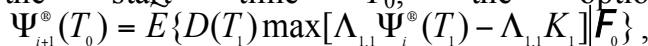

according to the fundamental theory of asset pricing (Baxter and Runie, 1996), where $F_{0}$ denotes the information available at time $T_{0}$ from the asset price.

$$
\begin{aligned}
& \Psi_{i+1}^{\circledast}\left(T_{0}\right)=\widetilde{E}\left\{D\left(T_{1}\right) \Lambda_{1,1} \Psi_{i}^{\circledast}\left(T_{1}\right) 1_{\left\{\hat{H}_{1}, \Psi_{i}^{\oplus},\left(T_{1}\right)>\Lambda_{1}, K_{1}\right.}\right\} \\
& -\widetilde{E}\left\{D\left(T_{1}\right) \Lambda_{1,1} K_{1} 1_{\left\{1, \Psi_{i}^{(\theta)}\left(T_{1}\right)>\Lambda_{1}, K_{1}\right.}\right\} \\
& =\Lambda_{1,1} \widetilde{E}\left\{\Lambda_{i+1,2} S_{1} \dot{\mathbf{I}}_{i}\left\{\left[\Lambda_{i+1, g+1} a_{i, g,{ }^{*} 1}^{\circledR}\right\rfloor_{\times 1} ;\left[\hat{\rho}_{g, h,{ }^{*} 1}\right]_{\times i}\right\}_{\left\{\Lambda_{1,1} \Psi_{i}^{\circledast}\left(T_{1}\right)>\Lambda_{1,1} K_{1}\right\}},\right.
\end{aligned}
$$

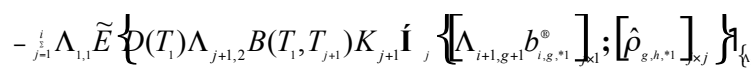

$$
\begin{aligned}
& -\Lambda_{1,1} B\left(0, T_{1}\right) K_{1} \widetilde{E}\left\{\frac{D\left(T_{1}\right)}{B\left(0, T_{1}\right)} 1_{\left\{\left\{_{\left.1,1, w_{i}^{m}\left(T_{1}\right)>\Lambda_{1}, K_{1}\right\}}\right\}\right.}\right\} \\
& \equiv \widetilde{\Psi}_{i+1,1}^{\circledast}-\widetilde{\Psi}_{i+1,2}^{\circledast}-\widetilde{\Psi}_{i+1,3}^{\circledast} \\
& \Psi_{i+1,1}^{\otimes}\left(T_{0}\right)=
\end{aligned}
$$$$
\left.\Lambda_{i+1,1} S_{0} \widetilde{E}\left\{\frac{D\left(T_{1}\right) S_{1}}{S_{0}} \mathbf{I}_{i}\left\{\left[\Lambda_{i+1, g+1} a_{i, g, *_{1}}^{\circledast}\right]_{\times 1} ;\left[\hat{\rho}_{g, h, *_{1} 1}\right]_{\times i}\right\}\right\}_{\left\{1, \Psi_{i}^{\otimes}\left(T_{1}\right)>\Lambda_{1,1} K_{1}\right\}}\right\}
$$

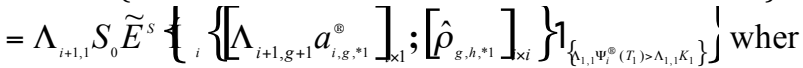$$
\text { e } \widetilde{E}^{s}[\cdot] \text { is the expectation operator under } \widetilde{\boldsymbol{P}}^{s} \text {. }
$$

$$
\text { According to (2.2) and (2.3), }
$$

$F\left(T_{0}, T_{1}, T_{g+1}\right)=F\left(T_{0}, T_{0}, T_{g+1}\right) e^{\left\{\frac{1}{2} \sigma_{1}^{2} \tau_{1}+\sigma_{1} \tilde{T}_{T_{1}}\right\}}$. Applying the last result, it could be should that

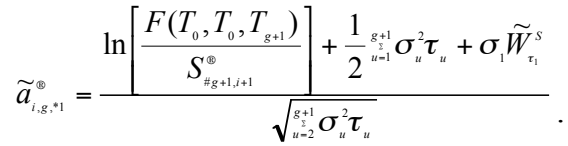

$$
\begin{aligned}
& \stackrel{d}{=} \frac{a_{i+1, g+1}^{\circledast}+z_{12} \rho_{1, g+1}}{\sqrt{1-\rho_{1, g+1}^{2}}} \equiv \bar{a}_{i, g, p}^{\circledast}, \forall 1 \leq g \leq i
\end{aligned}
$$

$z_{12}$ is the standard normal random variable.

$\Psi_{i+1,1}^{\circledast}\left(T_{0}\right)=\Lambda_{i+1,1} S_{0} \Lambda_{i+1,1} \int_{-a_{i+1,1}^{\infty}}^{\Lambda_{i+1,1} \infty} \frac{1}{\sqrt{2 \pi}} e^{-\frac{1}{2} z_{2}^{2}} \mathbf{I}_{i}\left\{\left[\Lambda_{i+1, g+1} \bar{a}_{i, g,{ }^{*} 1}^{\circledast}\right]_{\times 1} ;\left[\hat{\rho}_{g, h,{ }^{*} 1}\right]_{\times i}\right\}_{z_{12}}$

Note that the lower limit of the integration is also changed by (2.3).

Denote $z_{13}=-\Lambda_{i+1,1} z_{12}$, hence $\tilde{\Psi}_{i+1,1}^{\circledast}=\Lambda_{i+1,1} S_{0}$

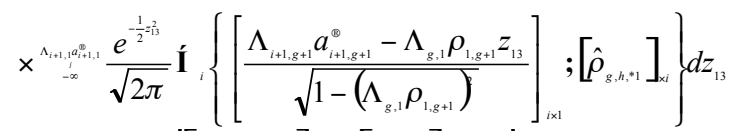
$=\Lambda_{i+1,1} S_{0} \mathbf{N}_{i+1}\left\{\left[\Lambda_{i+1, g} a_{i+1, g}^{\circledast}\right]_{i+1) \times 1} ;\left[H_{0, g, h}\right]_{i+1 \times(i+1)}\right\}$

The last equation is derived by Curnow \& Dunnett (1962) and the following is to exhibit that

$$
\left[H_{0, g, h}\right]_{t+1) \times(i+1)}=\left[\rho_{g, h}\right]_{+1) \times(i+1)} \text {. }
$$

According to Curnow \& Dunnett (1962), $H_{0,1,1}=1$; $H_{0,1, \mathrm{~g}}=\Lambda_{h-1,1} \rho_{1, h}, \forall 2 \leq g \leq i+1 ; H_{0, \mathrm{~g}, \mathrm{~h}}=H_{0, \mathrm{~h}, \mathrm{~g}} ; H_{0, \mathrm{~g}, \mathrm{~g}}=1$, $\forall 2 \leq g \leq i+1 . \quad \forall 2 \leq g<h \leq i+1$,

$$
\begin{aligned}
H_{0, g, h} & =\Lambda_{g-1,1} \rho_{1, g} \Lambda_{h-1,1} \rho_{1, h} \\
& +\sqrt{1-\left(\Lambda_{g-1,1} \rho_{1, g}\right)^{2}} \sqrt{1-\left(\Lambda_{h-1,1} \rho_{1, h}\right)^{2}} \hat{\rho}_{g-1, h-1, x_{1}} \\
& =\Lambda_{h-1, g} \frac{\sqrt{\frac{g}{\sum_{u=1}^{2} \sigma_{u}^{2} \tau_{u}}}}{\sqrt{\sum_{u=1}^{h} \sigma_{u}^{2} \tau_{u}}}=\Lambda_{h-1, g} \rho_{g, h}=\hat{\rho}_{g, h} .
\end{aligned}
$$

According to the above statements,

$$
\begin{aligned}
& \left.\left\lfloor H_{0, g, h}\right\rfloor_{i+1) \times(i+1)}=\rho_{g, h}\right\rfloor_{i+1) \times(i+1)} \text { and } \\
& \tilde{\Psi}_{i+1,1}^{\circledast}=\Lambda_{i+1,1} S_{0} \mathbf{N}_{i+1}\left\{\left[\Lambda_{i+1, g} a_{i+1, g}^{\circledast}\right]_{i+1) \times 1} ;\left[\hat{\rho}_{g, h}\right]_{i+1) \times(i+1)}\right\} .
\end{aligned}
$$

The $\tilde{\Psi}_{i+1,2}^{\circledast}$ and $\tilde{\Psi}_{i+1,3}^{\circledast}$ can be derived under the 1

$T$-forward measure $\widetilde{\boldsymbol{P}}^{T}$ with the corresponding expectation operator $\widetilde{E}^{T}[\cdot]$.

$$
\begin{aligned}
& \widetilde{\Psi}_{i+1,2}^{\circledast}={ }_{j=1}^{i} \Lambda_{j+1,1} K_{j+1} B\left(T_{0}, T_{j+1}\right) \\
& \times \widetilde{E}\left\{\frac{D\left(T_{1}\right)}{B\left(0, T_{1}\right)} \dot{\mathbf{I}}_{j}\left\{\left[\Lambda_{i+1, g+1} b_{i, g, *_{1}}^{\circledast}\right]_{\times 1} ;\left[\hat{\rho}_{g, h, *_{1}}\right]_{\times j}\right\}_{\left\{\left\{_{1,1, \Psi_{i}^{\oplus}\left(T_{1}\right)>\Lambda_{1}, K_{1}}\right\}\right.}\right\} \\
& ={ }_{j=1}^{i} \Lambda_{j+1,1} K_{j+1} B\left(T_{0}, T_{j+1}\right)
\end{aligned}
$$

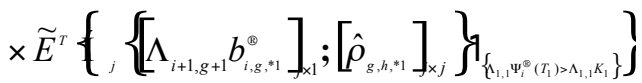

Substitute the (2.2) into the above equation and apply the similar way of $\tilde{\Psi}_{i+1,1}^{\circledast}$, it can be derived that

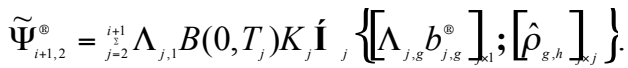

$\tilde{\Psi}_{i+1,3}^{\circledast} \quad$ can be derived by the same method. $\widetilde{\Psi}_{i+1,3}^{\circledast}=\Lambda_{1,1} B\left(0, T_{1}\right) K_{1} \mathbf{N}_{1}\left\{\Lambda_{i+1,1} b_{i+1,1}^{\circledast}\right\}$. Consequently, $\Psi_{i+1}^{\circledast}\left(T_{0}\right)$ is proved. Q.E.D.

\section{The Milestone Project Valuation (MPV)}

This section proposes the Milestone Projection Valuation (MPV) method for the multi-stage projects. The projects that set some critical milestones which should be achieved sequentially are call milestone projects. The milestone projects are failed if any one of the serial milestones is not completed. The milestone projects are very common in real situation, including R\&D management, manufactures, etc. Recently, the popular real option approach is applied for flexible consideration and reasonable explanation. Under the framework of financial option theory, the real option approach decomposes the project valuation as several parameters, including the present value, costs, time to maturity, value uncertainty (volatility) and interest rate. Most of the existing real option studies for the multi-stage milestone project valuations use one-fold options, while the others apply multi-fold options under the assumption of constant parameter through whole the processes (Casimon et al., 2004). However, the parameters often change due to the milestone completion and the project values will be misestimated if parameters are assumed constant through all the time. The one-fold real option approach for multi-stage project is even inadequate.

Based on the results of SCOs (2.1), this paper proposes a method called Milestone Projection Valuation (MPV) for multi-stage project valuation. Each milestone completion has the choice to enter the next stage or not, and the sequential project milestone can be viewed as the sequential compound CALL options. The MPV method adopts the results of SCOs and the project is regarded as the corresponding stock in SCOs. Under the same denotations as Theorem 1, the MPV valuation formula is expressed as 


$$
\begin{aligned}
& M P V_{i}^{\oplus}\left(T_{0}\right)=S_{0} \mathbf{I}_{i}\left\{\left[a_{i, g}^{\circledast}\right]_{i} ;\left[\rho_{g, h}\right]_{1}\right\} \\
& -{ }_{j=1}^{i} B\left(T_{0}, T_{j}\right) K_{j} \dot{\mathbf{I}}_{j} \quad\left\{\left[b_{i, g}^{\infty}\right]_{x 1} ;\left[\rho_{g, h}\right]_{x j}\right\}
\end{aligned}
$$

, where the strikes represent the cost at different stages; the volatilities come from the project value fluctuation and the dividend rates are replaced as the depression rates. The option features $\left(\Lambda_{i g}\right)$ equal one (for any $i, g$ ) due to the underlying compound calls, hence disappear in the MPV pricing formula.

Compared with the literatures, the MPV not only applies the multi-fold compound option theory, but also allows the parameters piece-constant varying with the distinct stages. The different parameters of different stages can adapt to the change of project nature after the milestone completion. More phenomena can be discovered from the parameter comparisons. Under the MPV model, the implicit "valuation experience" is decomposed as parameters.

The new drug applications (NDAs) may be the most famous and significant milestone projects. Under the consideration of human health, the NDAs are the well-regulated including the stages of pre-clinical trial, phase 1, phase 2, phase 3 and approval phase. Each stage has a definitive milestone. The time- and cost-consuming NDAs are the cores of the pharmaceutical companies because the R\&D results from NDAs dominate their future! The MPV model can enhance the NDAs valuation under a more reasonable framework and improve the R\&D management of these companies.

\section{Conclusion}

The puts/calls-alternating sequential compound options (SCOs) with random interest rate and fold-wise constant parameters are proposed in this study. Based on the results, the Milestone Projection Valuation (MPV) method is proposed for multi-stage project valuation.

Traditional compound options are just either puts/calls-alternating 2-fold compound options or multi-fold sequential compound call without puts/calls-alternating. Seldom fold-wise differences nor the random interest rate are taken into consideration. The SCOs with random interest rate presented in this paper have the following specialties. First of all, the multi-fold SCOs with arbitrary fold feature assignments as puts or calls can enhance the compound options usage far beyond the traditional sequential compound calls. Second, the parameters (interest rate, volatility) often vary with time or folds due to different characteristics. The presented SCOs formula enables random interest rate and volatility change fold-wise to capture the "sequential" features. The third is that the arbitrary fold number of SCOs can be formed.

The SCOs not only generalized the contributions of Black-Scholes (1973), Geske (1979) and Thomassen \& Van Wouwe (2001) to put/call alternating multi-fold compound options, but can be evaluated by linear combination of the stock and strike prices weighted by different variate normal integrations. Corresponding to intuitions, the SCOs seem as the multi-dimensional options extending from Black-Scholes (1973) and Geske (1979). The risk-neutral method enriches the SCOs pricing formula derivation with financial implications.

The SCOs can enhance and broaden the usages of compound option in real option and financial derivative fields. The multiple interacting options incorporating different type real options sophisticatedly can be evaluated by aggregation of various SCOs.

The MPV is designed for multi-stage project valuation. The MPV method adopts the results of SCO and the project is regarded as the corresponding stock in SCO. Compared with the literatures, the MPV not only applies the multi-fold compound option theory, but also allows the parameters piece-constant varying with the distinct stages. The different parameters of different stages can adapt to the change of project nature after the milestone completion. More phenomena can be discovered from the parameter comparisons. Under the MPV model, the implicit "valuation experience" is decomposed as parameters.

\section{References}

[1] Baxter, M. \& Runie, A. (1996), Financial Calculus: An Introduction to Derivative Pricing, Cambridge University Press.

[2] Bickel, P. J. and Doksum, K. A. (2001), Mathematical Statistics: Basic Ideas and Selected Topics, Vol.1, Second edition, Prentice Hall.

[3] Black, F. \& Scholes, M. (1973), "The Pricing of Options and Corporate Liabilities", Journal of Political Economy, vol. 81, p.637-657.

[4] Carr, P. (1988), "The Valuation of Sequential Exchange Opportunities", Journal of Finance, 43(5)1235-1256.

[5] Casimon, D., Engelen, P. J., Thomassen, L. \& Van Wouwe, M. (2004), "The Valuation of a NDA using a 6-fold compound option", Research Policy, vol.. 33, p.41-51.

[6] Chen, S. N. (2002), Financial Engineering , Hua-Tai.

[7] Chen, R. R. (2003), "The Extended Geske-Johnson Model and its Consistency with Reduced Form Models", working paper, Rutgers Business School, Rutgers University.

[8] Curnow, R. N. \& Dunnett, C. W. (1962), "The numerical evaluation of certain multivariate normal integrals", Annals of Mathematical Statistics, 33(2)571-579.

[9] Elettra, A. \& Rossella, A. (2003), "A generalization of Gesek formula for compound options", Mathematical Social Sciences, vol.45, p.75-82.

[10]Geske, R. (1979), "The Valuation of Compound Options", Journal of Financial Economics, 7(1)63-81

[11] Geske, R. \& Johnson, H. E. (1984a), "The American Put Option Valued Analytically", 39(5)1511-1524.

[12] Geske, R. \& Johnson, H. E. (1984b), "The Valuation of Corporate Liabilities as Compound Options: A Correction", 39(5)1511-1524.

[13] Lajeri-Chaherli, F. (2002), "A note on the Valuation of Compound Options", Journal of Futures Markets, 22(11)1103-1115.

[14] Lee, M.-Y., Yeh, F.-B \& Chen, A.-P.(2006), "Sequential Compound Options Pricing and Sensitivity Analysis", working paper.

[15] Musiela, M. \& Rutkowski, M. (1998), Martingale Methods in Financial Modelling, Springer.

[16]Remer, S., Ang, S.H. \& Baden-Fuller, C. (2001), "Dealing with uncertainties in the biotechnology industry: The use of real options reasoning", Journal of Commercial Biotechnology, 8(2)95-105.

[17] Rubinstein, M., "Options for the undecided", From Black Scholes to Black Holes, p.187-189, Risk Publication.

[18] Schroder, M. (1989), "A reduction method applicable to compound option formulas", Management Science, 35(7)823-827.

[19] Selby, M. J. P. \& Hogdes, S. D. (1987), "On the evaluation of compound options", Management Science, 33(3)347-355.

[20] Shreve, S. E. (2004), Stochastic Calculus for Finance II : Continuous-Time Models, Springer.

[21] Thomassen, L. \& Van Wouwe, M (2001), "The n-fold compound option", Research Paper 2001-041, University of Antwerp, Department of Mathematics and Statistics, $16 \mathrm{p}$.

[22] Thomassen, L. \& Van Wouwe, M (2002), "A Sensitivity analysis for the n-fold compound option", Research Paper 2002-014, University of Antwerp, Department of Mathematics and Statistics, $25 \mathrm{p}$.

[23] Trigeorgis, L. (1993), "The nature of option interactions and the valuation of investments with multiple real options", Journal of Financial and Quantitative analysis, 28(1)1-20.

[24] Trigeorgis, L. (1996), Real Options: Managerial Flexibility and Strategy in Resource Allocation, M.I.T. Press. 